\title{
Quantifying The Resolvability in CryoEM Structures
}

\author{
Wah Chiu and Greg Pintilie ${ }^{\mathrm{b}}$ \\ Department of Bioengineering, Stanford University, Stanford, CA 94305 \\ awahc@stanford.edu \\ bgregdp@gmail.com
}

Resolutions of many of the recent single particle CryoEM structures have been reported to range from 4.5 to $1.5 \AA$. The standard criterion of estimating resolution is based on the Fourier Shell correlation of two independent cryoEM maps. The complexity of cryoEM structures includes the existence of maps yielding multiple structures from a single specimen as well as non-uniform resolutions within a map [1].

Therefore, a single numerical value of resolution to describe the structure of a cryoEM map is rather inadequate. When a model is built from a cryoEM map, it has not only to be stereochemically compatible but also to match with the observed densities [2]. Due to the issue of variable resolvability in different parts of the maps often seen in the loops or flexible regions, and also of low or negative density value observed in the negative charged amino acids, it is useful to have scoring parameters to reflect the resolvability of the backbone, side chains or atoms [3]. Such parameters should be submitted to Protein Data Bank to provide clear guidance to the readers about the structure as how to evaluate its biochemical and functional interpretations.

\section{References}

[1] Zhang, K., et al., Proc Natl Acad Sci U S A (2019) 116, 6800-6805.

[2] Hryc, C.F., et al., Proc Natl Acad Sci U S A (2017) 114, 3103-3108.

[2] Pintilie, G. and Chiu, W. J Struct Biol (2018) 204, 564-571. 